INR-820/93

OUT $-4102-45$

\title{
Connections between deep-inelastic and annihilation processes at next-to-next-to-leading order and beyond ${ }^{1}$
}

\author{
D. J. Broadhurst ${ }^{2}$ \\ Physics Department, Open University, \\ Milton Keynes, MK7 6AA, UK \\ A. L. Kataev ${ }^{3}$ \\ Institute for Nuclear Research of the Academy of Sciences of Russia, \\ 117312 Moscow, Russia
}

\begin{abstract}
We have discovered 7 intimate connections between the published results for the radiative corrections, $C_{\mathrm{K}}$, to the Gross-Llewellyn Smith (GLS) sum rule, in deepinelastic lepton scattering, and the radiative corrections, $C_{\mathrm{R}}$, to the Adler function of the flavour-singlet vector current, in $\mathrm{e}^{+} \mathrm{e}^{-}$annihilation. These include a surprising relation between the scheme-independent single-electron-loop contributions to the 4-loop QED $\beta$-function and the zero-fermion-loop abelian terms in the 3-loop GLS sum rule. The combined effect of all 7 relations is to give the factorization of the 2 -loop $\beta$-function in

$$
\Delta_{\mathrm{S}} \equiv C_{\mathrm{K}} C_{\mathrm{R}}-1=\frac{\beta\left(\bar{a}_{\mathrm{s}}\right)}{\bar{a}_{\mathrm{s}}}\left\{S_{1} C_{\mathrm{F}} \bar{a}_{\mathrm{s}}+\left[S_{2} T_{\mathrm{F}} N_{\mathrm{F}}+S_{\mathrm{A}} C_{\mathrm{A}}+S_{\mathrm{F}} C_{\mathrm{F}}\right] C_{\mathrm{F}} \bar{a}_{\mathrm{s}}^{2}\right\}+O\left(\bar{a}_{\mathrm{s}}^{4}\right),
$$

where $\bar{a}_{\mathrm{s}}=\alpha_{\mathrm{s}}\left(\mu^{2}=Q^{2}\right) / 4 \pi$ is the $\overline{\mathrm{MS}}$ coupling of an arbitrary colour gauge theory, and

$$
S_{1}=-\frac{21}{2}+12 \zeta_{3} ; \quad S_{2}=\frac{326}{3}-\frac{304}{3} \zeta_{3} ; \quad S_{\mathrm{A}}=-\frac{629}{2}+\frac{884}{3} \zeta_{3} ; \quad S_{\mathrm{F}}=\frac{397}{6}+136 \zeta_{3}-240 \zeta_{5}
$$

specify the sole content of $C_{\mathrm{K}}$ that is not already encoded in $C_{\mathrm{R}}$ and $\beta\left(\bar{a}_{\mathrm{s}}\right)=Q^{2} \mathrm{~d} \bar{a}_{\mathrm{s}} / \mathrm{d} Q^{2}$ at $O\left(\bar{a}_{\mathrm{s}}^{3}\right)$. The same result is obtained by combining the radiative corrections to Bjorken's polarized sum rule with those for the Adler function of the non-singlet axial current. We suggest possible origins of $\beta$ in the 'Crewther discrepancy', $\Delta_{\mathrm{S}}$, and determine $\Delta_{\mathrm{S}} /\left(\beta\left(\bar{a}_{\mathrm{S}}\right) / \bar{a}_{\mathrm{S}}\right)$, to all orders in $N_{\mathrm{F}} \bar{a}_{\mathrm{S}}$, in the large- $N_{\mathrm{F}}$ limit, obtaining the entire series of coefficients of which $S_{1}$ and $S_{2}$ are merely the first two members.
\end{abstract}

INR-820/93

OUT-4102-45

1) In memoriam Sergei Grigorievich Gorishny, 1958-1988

2) D.Broadhurst@open.ac.uk

3) Kataev@inucres.msk.su 


\section{Introduction}

In 1972, Crewther [1] related three fundamental constants of an arbitrary parton model: the anomalous constant $S$, associated with the amplitude for $\pi^{0} \rightarrow \gamma \gamma$ decay [2]; the coefficient $K$ in Bjorken's sum rule for polarized deep-inelastic electron scattering [3]; and the constant $R^{\prime}$ in the annihilation channel, giving the asymptotic value of the Adler function [4] for the correlator of the iso-vector axial current. His non-perturbative derivation relied on conformal and chiral invariance of the leading short-distance singularity, with coefficient $S$, in the operator product expansion (OPE) of the 3-point function $A V V$, for $\pi_{0}$ decay, where $A=J_{5}^{\mu}$ is the iso-vector axial current and $V=J_{\mathrm{EM}}^{\mu}$ is the electromagnetic current. To obtain $3 S=K R^{\prime}$ [1], one first takes the OPE of the 2-point function $V V$, in which one encounters the axial current, $A$, with the coefficient $K$ of Bjorken's polarized sum rule. Then one obtains $R^{\prime}$ in the leading term of the resultant OPE of the $A A$-correlator, corresponding to the Adler function of the iso-vector axial current.

The relation $3 S=K R^{\prime}$ is, necessarily, satisfied by the standard quark-parton model, which gives $S=\frac{1}{2}, K=1, R^{\prime}=\frac{3}{2}$, for an iso-doublet of $u$ and $d$ quarks, each having $N_{\mathrm{C}}=3$ colours. The chiral symmetry of the quark-parton model means that $K$ also occurs in the Gross-Llewellyn Smith (GLS) sum rule [5] of deep-inelastic neutrino scattering, corresponding to the coefficient of the vector current in the vector-axial correlator. Invoking both chiral symmetry and $\mathrm{SU}(3)$-flavour symmetry [1], one obtains $R^{\prime}=\frac{3}{4} R$, where $R=2$ corresponds to the Adler function of the $V V$-correlator, giving the quark-parton model prediction for $\mathrm{e}^{+} \mathrm{e}^{-}$annihilation (below the charm threshold).

It is not at all clear what the theoretical status and phenomenological consequences of the Crewther connection might be in QCD, where radiative corrections to the naive quark-parton model give a dependence on the running coupling $\bar{a}_{\mathrm{s}}=\alpha_{\mathrm{s}}\left(\mu^{2}=Q^{2}\right) / 4 \pi$ that is appreciable at presently accessible values of $-q^{2} \equiv Q^{2}$. In this paper we study radiative corrections to deep-inelastic [6] and annihilation [7] processes, at next-to-next-to-leading order (NNLO) in the $\overline{\mathrm{MS}}$ scheme, discovering that they are intimately connected, in a manner that is profoundly related to the Crewther connection [1].

\section{Deep-inelastic and annihilation results at NNLO}

In deep-inelastic lepton scattering, radiative corrections to the GLS sum rule [5],

$$
\frac{1}{2} \int_{0}^{1} \mathrm{~d} x F_{3}^{\bar{\nu} \mathrm{p}+\nu \mathrm{p}}\left(x, Q^{2}\right)=3 C_{\mathrm{GLS}}\left(\bar{a}_{\mathrm{s}}\right)
$$

and to Bjorken's polarized sum rule [3],

$$
\int_{0}^{1} \mathrm{~d} x g_{1}^{\mathrm{ep}-\mathrm{en}}\left(x, Q^{2}\right)=\frac{1}{3}\left|\frac{g_{\mathrm{A}}}{g_{\mathrm{V}}}\right| C_{\mathrm{Bjp}}\left(\bar{a}_{\mathrm{s}}\right),
$$

have been obtained [6] to NNLO in the $\overline{\mathrm{MS}}$ scheme, where the dependence on $Q^{2}=-q^{2}$ is absorbed, at large $Q^{2}$, into the coupling $\bar{a}_{\mathrm{s}}=\alpha_{\mathrm{s}}\left(\mu^{2}=Q^{2}\right) / 4 \pi$. A difference between

$C_{\mathrm{GLS}}$ and $C_{\mathrm{Bjp}}$ is first encountered at $O\left(\bar{a}_{\mathrm{s}}^{3}\right)$, where so called 'light-by-light-type' diagrams contribute to $C_{\mathrm{GLS}}$, but not to $C_{\mathrm{Bjp}}$. 
In annihilation, at large $Q^{2}$, the Adler functions $D_{\mathrm{EM}}^{\mathrm{V}}$ and $D_{\mathrm{NS}}^{\mathrm{A}}$ have been calculated [7] at NNLO, again in the $\overline{\mathrm{MS}}$ scheme, for the electromagnetic current $J_{\mathrm{EM}}^{\mu}=\sum_{f} Q_{f} \bar{\psi}_{f} \gamma^{\mu} \psi_{f}$, where the sum is over $N_{\mathrm{F}}$ flavours of quark, with charges $Q_{f}$, and for the non-singlet axial current $J_{5}^{\mu}=\bar{\psi}_{i} \gamma^{\mu} \gamma_{5} \psi_{j}$, where $i$ and $j$ are different quark flavours. In [7] it was assumed that $D_{\mathrm{NS}}^{\mathrm{A}}$, relevant to $\tau$ decay, is identical to the Adler function $D_{\mathrm{NS}}^{\mathrm{V}}$, of the nonsinglet vector current, whose radiative corrections are obtained by omitting the NNLO light-by-light-type terms that contribute to $D_{\mathrm{EM}}^{\mathrm{V}}$. Analytic continuation of $D_{\mathrm{EM}}^{\mathrm{V}}$ and $D_{\mathrm{NS}}^{\mathrm{A}}$ to the time-like region yields contributions to the processes $\mathrm{e}^{+} \mathrm{e}^{-} \rightarrow$ hadrons and $\tau \rightarrow \nu_{\tau}+$ hadrons. Note that the correlator of the singlet axial current, related to $Z^{0} \rightarrow$ hadrons, receives anomalous contributions from diagrams with gluons in the intermediate state, considered in [8, 9].

To investigate the possibility of a perturbative Crewther connection, in colour gauge theories, we study the radiative corrections

$$
\begin{aligned}
& C_{\mathrm{K}} \equiv C_{\mathrm{GLS}}\left(\bar{a}_{\mathrm{s}}\right)=1-3 C_{\mathrm{F}} \bar{a}_{\mathrm{s}}+O\left(\bar{a}_{\mathrm{s}}^{2}\right), \\
& C_{\mathrm{R}} \equiv \frac{D^{\mathrm{V}}\left(\bar{a}_{\mathrm{s}}\right)}{N_{\mathrm{F}} N_{\mathrm{C}}}=1+3 C_{\mathrm{F}} \bar{a}_{\mathrm{s}}+O\left(\bar{a}_{\mathrm{s}}^{2}\right), \quad D^{\mathrm{V}} \equiv-12 \pi^{2} Q^{2} \frac{\mathrm{d} \Pi^{\mathrm{V}}}{\mathrm{d} Q^{2}},
\end{aligned}
$$

to the GLS sum rule (11) and the Adler function, $D^{\mathrm{V}}$, of the correlator $\left(q_{\mu} q_{\nu}-q^{2} g_{\mu \nu}\right) \Pi^{\mathrm{V}}$ of the flavour-singlet vector current $J_{\mu}=\sum_{f} \bar{\psi}_{f} \gamma_{\mu} \psi_{f}$, with $N_{\mathrm{F}}$ active quark flavours.

The NNLO results for $C_{\mathrm{K}}$ [6] and $C_{\mathrm{R}}$ [0], in the $\overline{\mathrm{MS}}$ scheme, are given in Table 1, for an arbitrary colour gauge group. (The colour factors take the values $T_{\mathrm{F}}=\frac{1}{2}, C_{\mathrm{A}}=N_{\mathrm{C}}=3$, $C_{\mathrm{F}}=\frac{4}{3}, d_{a b c}^{2}=\frac{40}{3}$, in the particular case of QCD.) To obtain the radiative corrections (画), to the flavour-singlet Adler function $D^{\mathrm{V}}$, one has merely to give the quarks equal charges, $Q_{f}=$ constant, in $D_{\mathrm{EM}}^{\mathrm{V}}$, corresponding to setting $\left(\sum_{f} Q_{f}\right)^{2}=N_{\mathrm{F}} \sum_{f} Q_{f}^{2}$ in the results of [7]. (Note that we consider $D^{\mathrm{V}}$ at large space-like $Q^{2}=-q^{2}$ and hence omit the $\pi^{2}$ terms of Eq. (12) of [7], which result from analytic continuation to the time-like region.) The NNLO radiative corrections to $C_{\mathrm{Bjp}}$ are obtained by dropping light-by-light-type terms, proportional to $d_{a b c}^{2}$, from $C_{\mathrm{K}} \equiv C_{\mathrm{GLS}}$; the corrections to $D_{\mathrm{NS}}^{\mathrm{A}} / N_{\mathrm{C}}=D_{\mathrm{NS}}^{\mathrm{V}} / N_{\mathrm{C}}$ are obtained by dropping them from $C_{\mathrm{R}} \equiv D^{\mathrm{V}} / N_{\mathrm{F}} N_{\mathrm{C}}$.

At first glance, one is tempted to conclude from [四] that the radiative corrections (3. (3) might give a product, $C_{\mathrm{K}} C_{\mathrm{R}}$, that is free of radiative corrections, in the spirit of the norenormalization theorem [10] for the axial anomaly that determines $\pi_{0}$ decay in the zeromass limit. However, the NLO corrections to $C_{\mathrm{K}}$ [11] and $C_{\mathrm{R}}$ [12] give $C_{\mathrm{K}} C_{\mathrm{R}} \neq 1$, when one absorbs the $\alpha_{\mathrm{s}}^{2} \ln \left(Q^{2} / \mu^{2}\right)$ term of each process in the $\overline{\mathrm{MS}}$ coupling $\bar{a}_{\mathrm{s}}=\alpha_{\mathrm{s}}\left(\mu^{2}=Q^{2}\right) / 4 \pi$. The recent availability of highly non-trivial NNLO results, for both $C_{\mathrm{K}}$ [6] and $C_{\mathrm{R}}$ [7], prompted us to study the 'Crewther discrepancy' $\Delta_{\mathrm{S}} \equiv\left(C_{\mathrm{K}} C_{\mathrm{R}}-1\right)$, at $O\left(\bar{a}_{\mathrm{s}}^{3}\right)$.

\section{Anatomy of a discovery}

After much investigation of Table 1, we discovered the following remarkable relation between the $\overline{\mathrm{MS}}$ results of [6, 7], for any colour gauge theory, renormalized at $\mu^{2}=Q^{2}$ :

$$
\Delta_{\mathrm{S}} \equiv C_{\mathrm{K}} C_{\mathrm{R}}-1=\frac{\beta\left(\bar{a}_{\mathrm{s}}\right)}{\bar{a}_{\mathrm{s}}}\left\{S_{1} C_{\mathrm{F}} \bar{a}_{\mathrm{s}}+\left[S_{2} T_{\mathrm{F}} N_{\mathrm{F}}+S_{\mathrm{A}} C_{\mathrm{A}}+S_{\mathrm{F}} C_{\mathrm{F}}\right] C_{\mathrm{F}} \bar{a}_{\mathrm{s}}^{2}\right\}+O\left(\bar{a}_{\mathrm{s}}^{4}\right),
$$


where $\beta\left(\bar{a}_{\mathrm{s}}\right) \equiv Q^{2} \mathrm{~d} \bar{a}_{\mathrm{s}} / \mathrm{d} Q^{2}=\bar{a}_{\mathrm{s}} \sum_{n \geq 1} \beta_{n} \bar{a}_{\mathrm{s}}^{n}$, and

$$
S_{1}=-\frac{21}{2}+12 \zeta_{3} ; \quad S_{2}=\frac{326}{3}-\frac{304}{3} \zeta_{3} ; \quad S_{\mathrm{A}}=-\frac{629}{2}+\frac{884}{3} \zeta_{3} ; \quad S_{\mathrm{F}}=\frac{397}{6}+136 \zeta_{3}-240 \zeta_{5}
$$

specify the sole NNLO content of $C_{\mathrm{K}}$ that is not derivable from $C_{\mathrm{R}}$ and from the coefficients

$$
\beta_{1}=-\frac{11}{3} C_{\mathrm{A}}+\frac{4}{3} T_{\mathrm{F}} N_{\mathrm{F}} ; \quad \beta_{2}=-\frac{34}{3} C_{\mathrm{A}}^{2}+\frac{20}{3} C_{\mathrm{A}} T_{\mathrm{F}} N_{\mathrm{F}}+4 C_{\mathrm{F}} T_{\mathrm{F}} N_{\mathrm{F}}
$$

of the two-loop $\beta$-function. The same result is obtained by combining the NNLO corrections to Bjorken's polarized sum rule (2) with those for the Adler function $D_{\mathrm{NS}}^{\mathrm{A}}$ of the non-singlet axial current, which differ from $C_{\mathrm{K}}$ and $C_{\mathrm{R}}$, respectively, merely by omitting $d_{a b c}^{2} \bar{a}_{\mathrm{s}}^{3}$ terms that cancel in (5).

Since $C_{\mathrm{K}}$ and $C_{\mathrm{R}}$, taken up to $O\left(\bar{a}_{\mathrm{s}}^{3}\right)$, each involve the 11 distinct colour factors of the terms $\left\{T_{n} \mid n=1,11\right\}$, defined in Table 1 , the existence of a relation of the form of (5) entails the following 'seven wonders' of the Crewther discrepancy $\Delta_{\mathrm{S}} \equiv\left(C_{\mathrm{K}} C_{\mathrm{R}}-1\right)$ :

1. The leading-order terms cancel in $\Delta_{S}$.

2. The NLO corrections give no $C_{\mathrm{F}}^{2} \bar{a}_{\mathrm{s}}^{2}$ term in $\Delta_{\mathrm{S}}$.

3. The NLO corrections give $C_{\mathrm{F}} C_{\mathrm{A}} \bar{a}_{\mathrm{s}}^{2}$ and $C_{\mathrm{F}} T_{\mathrm{F}} N_{\mathrm{F}} \bar{a}_{\mathrm{s}}^{2}$ terms in $\Delta_{\mathrm{S}}$ that are in the same ratio as the $C_{\mathrm{A}}$ and $T_{\mathrm{F}} N_{\mathrm{F}}$ terms in $\beta_{1}$.

4. The NNLO corrections give no $C_{\mathrm{F}}^{3} \bar{a}_{\mathrm{s}}^{3}$ term in $\Delta_{\mathrm{S}}$. This leads to the astonishing observation that the scheme-independent single-electron-loop contributions, $\beta_{\mathrm{QED}}^{[1]}$, to the QED $\beta$-function, are obtained, up to 4-loops, by taking the reciprocal of the zero-fermion-loop abelian terms in the 3-loop GLS result of [6], giving

$$
\beta_{\mathrm{QED}}^{[1]}(a)=\frac{\frac{4}{3} a^{2}}{1-3 a+\frac{21}{2} a^{2}-\frac{3}{2} a^{3}+O\left(a^{4}\right)}=\frac{4}{3} a^{2}+4 a^{3}-2 a^{4}-46 a^{5}+O\left(a^{6}\right),
$$

in precise agreement with [13]. Such is the power of relation (5).

5. The NNLO light-by-light-type terms of [6] and [7], involving $T_{11} \equiv \frac{N_{\mathrm{F}}}{N_{\mathrm{C}}} d_{a b c}^{2} \bar{a}_{\mathrm{s}}^{3}$, cancel in $\Delta_{\mathrm{S}}$ (taking equal quark charges in [7], to obtain the singlet Adler function $D^{\mathrm{V}}$ ).

6. The NNLO corrections in $\Delta_{\mathrm{S}}$ are expressible as the sum of multiples of the one-loop and two-loop contributions, $\beta_{1} \bar{a}_{\mathrm{s}}^{2}$ and $\beta_{2} \bar{a}_{\mathrm{s}}^{3}$, to the $\beta$-function.

7. At NNLO, $\beta_{2} \bar{a}_{\mathrm{s}}^{3}$ occurs in $\Delta_{\mathrm{S}}$ with the same coefficient that multiplies $\beta_{1} \bar{a}_{\mathrm{s}}^{2}$, at NLO, allowing one to factor out $\beta\left(\bar{a}_{\mathrm{s}}\right)$ in (5). Moreover, this factorization is independent of the momenta in the two processes: if one takes the GLS sum-rule results at a momentum transfer $-q^{2}=Q_{\mathrm{K}}^{2}$, and the Adler function $D^{\mathrm{V}}$ at $-q^{2}=Q_{\mathrm{R}}^{2}$, the factorization of (5) still occurs, with the replacements $\bar{a}_{\mathrm{s}} \rightarrow \alpha_{\mathrm{s}}\left(\mu^{2}=Q_{\mathrm{R}}^{2}\right) / 4 \pi$ and

$$
S_{1} \rightarrow S_{1}-3 \lambda ; S_{2} \rightarrow S_{2}+16 \lambda-4 \lambda^{2} ; S_{\mathrm{A}} \rightarrow S_{\mathrm{A}}-46 \lambda+11 \lambda^{2} ; S_{\mathrm{F}} \rightarrow S_{\mathrm{F}}+12 \lambda ;
$$

where $\lambda=\ln \left(Q_{\mathrm{K}}^{2} / Q_{\mathrm{R}}^{2}\right)$. 
The corresponding 7 relations between the coefficients of $C_{\mathrm{K}}=\sum_{n} k_{n} T_{n}+O\left(\bar{a}_{\mathrm{s}}^{4}\right)$ and $C_{\mathrm{R}}=\sum_{n} r_{n} T_{n}+O\left(\bar{a}_{\mathrm{s}}^{4}\right)$, given in Table 1, can be divided into two groups. Observations $1,2,4,5$, above, correspond to the 4 conditions

$$
0=k_{1}+r_{1}=k_{2}+r_{2}+k_{1} r_{1}=k_{5}+r_{5}+k_{1} r_{2}+r_{1} k_{2}=k_{11}+r_{11},
$$

which are required to ensure the absence of terms in $\Delta_{\mathrm{S}}$ that cannot occur in the factorization (5). Observations 3,6,7, above, correspond to the 3 remaining conditions

$$
\begin{aligned}
-\frac{3}{11}\left(k_{3}+r_{3}\right) & =\frac{3}{4}\left(k_{4}+r_{4}\right) \\
& =\frac{1}{7}\left(k_{7}+r_{7}\right)+\frac{11}{28}\left(k_{9}+r_{9}\right)+\frac{121}{112}\left(k_{10}+r_{10}\right) \\
& =\frac{1}{11}\left(k_{6}+r_{6}+k_{1} r_{3}+r_{1} k_{3}\right)+\frac{1}{4}\left(k_{8}+r_{8}+k_{1} r_{4}+r_{1} k_{4}\right),
\end{aligned}
$$

which relate equivalent ways of evaluating $S_{1}=-\frac{21}{2}+12 \zeta_{3}$, consistent with (5, (7). We invite any reader who may still doubt the significance of the factorization of the two-loop $\beta$-function in (5) to use the coefficients of Table 1 to verify that the 7 necessary conditions in $(10,11)$ are satisfied in a highly non-trivial manner.

We relate our discovery (5) to [1] by observing that it seems rather natural to obtain $C_{\mathrm{K}} C_{\mathrm{R}}=1$ at any fixed point, where $\beta=0$, since Crewther's assumptions of conformal and chiral invariance should hold in that scale-free limit. We note that the anomalous dimension of the pseudo-scalar operator $G^{\mu \nu} \widetilde{G}_{\mu \nu}$, in the anomalous divergence of the singlet axial current, is $-\beta\left(a_{\mathrm{s}}\right) / a_{\mathrm{s}}$ [14]. This makes it reasonable that $C_{\mathrm{K}} C_{\mathrm{R}}=1$, when $\beta=0$, corresponding to no renormalization of the anomaly at any fixed point, and hence suggesting that one may expect to find a Crewther discrepancy $\Delta_{\mathrm{S}} \equiv\left(C_{\mathrm{K}} C_{\mathrm{R}}-1\right) \propto \beta$. (See [14, 15] for recent studies of corrections to the one-loop axial anomaly equation.)

Though we cannot yet be sure that $\beta$ can be factored out of $\left(C_{\mathrm{K}} C_{\mathrm{R}}-1\right)$, beyond NNLO, it seems most likely to us that at any given order, $\bar{a}_{\mathrm{s}}^{N}$, one will encounter in $\Delta_{\mathrm{S}}$ only the coefficients $\left\{\beta_{n} \mid n<N\right\}$, multiplied by linear combinations of colour factors. We believe that it is the scale-dependent procedure of renormalization that modifies the naive result $\Delta_{\mathrm{S}}=\left(C_{\mathrm{K}} C_{\mathrm{R}}-1\right)=0$, suggested by the conformal arguments of [1] and the essentially one-loop nature of the anomaly [10]. Since the coefficients $\beta_{n}$ multiply all scale-dependent perturbative artefacts of the renormalization procedure, one expects them to occur in $\Delta_{\mathrm{S}}$. This does not, of itself, require that $\Delta_{\mathrm{S}} \propto \beta$. However, the existence of the relations (11), between the highly non-trivial coefficients of Table 1, may be taken as powerful circumstantial evidence in favour of this stronger hypothesis.

We leave these considerations for later work and now obtain all the $O\left(1 / N_{\mathrm{F}}\right)$ terms of

$$
\frac{C_{\mathrm{K}} C_{\mathrm{R}}-1}{\beta\left(\bar{a}_{\mathrm{s}}\right) / \bar{a}_{\mathrm{s}}}=\frac{C_{\mathrm{F}}}{T_{\mathrm{F}} N_{\mathrm{F}}} \sum_{n=1}^{\infty} S_{n}\left(T_{\mathrm{F}} N_{\mathrm{F}} \bar{a}_{\mathrm{s}}\right)^{n}+O\left(1 / N_{\mathrm{F}}^{2}\right)
$$

in the $\overline{\mathrm{MS}}$ scheme, taking the limit $N_{\mathrm{F}} \rightarrow \infty$, with $N_{\mathrm{F}} \bar{a}_{\mathrm{s}}$ fixed. In obtaining all the coefficients $S_{n}$, we provide an all-orders consistency test of the procedures of [6].

\section{All-orders results at large $N_{\mathrm{F}}$}

We follow [6, 9, 11, 14, 16, 17, 18] in defining an axial current, within the framework of dimensional regularization, by calculating Green functions of the renormalized non-singlet 
antisymmetric-tensor current

$$
A_{\kappa \lambda \mu} \equiv \frac{\mathrm{i}}{2} Z_{\mathrm{A}} \bar{\psi}_{i}\left(\gamma_{\kappa} \gamma_{\lambda} \gamma_{\mu}-\gamma_{\mu} \gamma_{\lambda} \gamma_{\kappa}\right) \psi_{j}, \quad Z_{\mathrm{A}}=1+\sum_{n=1}^{\infty} a_{\mathrm{s}}^{n} \sum_{p=0}^{n-1} \frac{Z_{n, p}}{\varepsilon^{p}}
$$

where $i$ and $j$ are different quark flavours, $d \equiv 4-2 \varepsilon$ is the spacetime dimension, $a_{\mathrm{s}}=\alpha_{\mathrm{s}} / 4 \pi$ is the $\overline{\mathrm{MS}}$ coupling, renormalized at scale $\mu$, and $Z_{\mathrm{A}}$ is a non-minimal renormalization constant, constructed so as to preserve chiral symmetry and to have vanishing anomalous dimension. The condition

$$
\frac{\mathrm{d} \ln Z_{\mathrm{A}}}{\mathrm{d} \ln \mu^{2}}=\left(-\varepsilon+\frac{\beta\left(a_{\mathrm{s}}\right)}{a_{\mathrm{s}}}\right) \frac{\mathrm{d} \ln Z_{\mathrm{A}}}{\mathrm{d} \ln a_{\mathrm{s}}}=O(\varepsilon)
$$

thus relates the singular terms in (13) to the finite terms, giving NNLO singular terms

$$
Z_{2,1}=\frac{1}{2} \beta_{1} Z_{1,0}, \quad Z_{3,2}=\frac{1}{3} \beta_{1}^{2} Z_{1,0}, \quad Z_{3,1}=\frac{1}{3} \beta_{2} Z_{1,0}+\frac{1}{6} \beta_{1}\left(Z_{1,0}^{2}+4 Z_{2,0}\right),
$$

in terms of $\beta_{1}$ and $\beta_{2}$, in (17), and the NLO finite terms [6, 14]

$$
Z_{1,0}=-4 C_{\mathrm{F}}, \quad Z_{2,0}=22 C_{\mathrm{F}}^{2}-\frac{107}{9} C_{\mathrm{F}} C_{\mathrm{A}}+\frac{4}{9} C_{\mathrm{F}} T_{\mathrm{F}} N_{\mathrm{F}} .
$$

The procedure for obtaining Green functions involving the non-singlet axial current is to combine the non-minimal renormalization of $(\sqrt{13})$ with the standard $\overline{\mathrm{MS}}$ renormalization of the bare coupling constant, $g_{0}$. One then subtracts any polynomial in the momenta that is singular at $\varepsilon=0$, and takes the limit $\varepsilon \rightarrow 0$. Thereafter, one multiplies by the appropriate number of 4-dimensional Levi-Civita tensors (one for each axial vertex), to obtain renormalized Green functions of the conventional 4-dimensional axial current, which may be written schematically as

$$
A_{\nu}=\bar{\psi}_{i} \gamma_{\nu} \gamma_{5} \psi_{j} \cong \frac{1}{3 !} \varepsilon_{\kappa \lambda \mu \nu} \lim _{d \rightarrow 4}\left[\frac{\mathrm{i}}{2} Z_{\mathrm{A}} \bar{\psi}_{i}\left(\gamma^{\kappa} \gamma^{\lambda} \gamma^{\mu}-\gamma^{\mu} \gamma^{\lambda} \gamma^{\kappa}\right) \psi_{j}\right]
$$

with the limit $d \rightarrow 4$ taken after all renormalization. Before renormalization, all reference to the Levi-Civita tensor, and hence to $\gamma_{5}$, is resolutely avoided. This enables covariant $d-$ dimensional calculation, albeit at the expense of large traces over $\gamma$-matrices. With traces involving only an even number of axial vertices, it is presumed [19 that all physical results are identical to those that would have been obtained by naively using the 4-dimensional identities $\gamma_{\mu} \gamma_{5}=-\gamma_{5} \gamma_{\mu}$ and $\gamma_{5}^{2}=1$. We now test this in an all-orders calculation.

\subsection{Annihilation processes}

The renormalized correlator of the vector current $V_{\mu} \equiv \bar{\psi}_{i} \gamma_{\mu} \psi_{j}$ may be written as

$$
\mathrm{i} \int \mathrm{d} x \mathrm{e}^{\mathrm{i} q \cdot x}\left\langle T\left\{V^{\mu}(x) V_{\nu}^{\dagger}(0)\right\}\right\rangle=-q^{2} g_{\nu}^{\mu} \Pi_{\mathrm{T}}^{\mathrm{V}}+q^{\mu} q_{\nu}\left(\Pi_{\mathrm{L}}^{\mathrm{V}}+\Pi_{\mathrm{T}}^{\mathrm{V}}\right)
$$

with $\Pi_{\mathrm{L}}^{\mathrm{V}}=0$, for massless quarks. The decomposition of the correlator of (13) may be written as

$$
\mathrm{i} \int \mathrm{d} x \mathrm{e}^{\mathrm{i} q \cdot x}\left\langle T\left\{A^{\alpha \beta \gamma}(x) A_{\kappa \lambda \mu}^{\dagger}(0)\right\}\right\rangle=-q^{2} G_{\kappa \lambda \mu}^{\alpha \beta \gamma} \Pi_{\mathrm{T}}^{\mathrm{A}}+G_{\kappa \lambda \mu \nu}^{\alpha \beta \gamma \delta} q^{\nu} q_{\delta}\left(\Pi_{\mathrm{L}}^{\mathrm{A}}+\Pi_{\mathrm{T}}^{\mathrm{A}}\right),
$$


with a tensor structure given by the determinants

$$
G_{\kappa \lambda \mu}^{\alpha \beta \gamma} \equiv\left|\begin{array}{ccc}
g_{\kappa}^{\alpha} & g_{\lambda}^{\alpha} & g_{\mu}^{\alpha} \\
g_{\kappa}^{\beta} & g_{\lambda}^{\beta} & g_{\mu}^{\beta} \\
g_{\kappa}^{\gamma} & g_{\lambda}^{\gamma} & g_{\mu}^{\gamma}
\end{array}\right|, \quad G_{\kappa \lambda \mu \nu}^{\alpha \beta \gamma \delta} \equiv\left|\begin{array}{cccc}
g_{\kappa}^{\alpha} & g_{\lambda}^{\alpha} & g_{\mu}^{\alpha} & g_{\nu}^{\alpha} \\
g_{\kappa}^{\beta} & g_{\lambda}^{\beta} & g_{\mu}^{\beta} & g_{\nu}^{\beta} \\
g_{\kappa}^{\gamma} & g_{\lambda}^{\gamma} & g_{\mu}^{\gamma} & g_{\nu}^{\gamma} \\
g_{\kappa}^{\delta} & g_{\lambda}^{\delta} & g_{\mu}^{\delta} & g_{\nu}^{\delta}
\end{array}\right| .
$$

For massless quarks, chiral symmetry requires that $\Pi_{\mathrm{L}}^{\mathrm{A}}$ and $\left(\Pi_{\mathrm{T}}^{\mathrm{A}}-\Pi_{\mathrm{T}}^{\mathrm{V}}\right)$ be constants. Note that they may be non-zero, since the non-minimal renormalization of (13), combined with minimal subtraction of infinities, may still leave chiral-symmetry-breaking finite terms in the renormalized expressions for divergent quantities. Only the subtraction-free nonsinglet Adler functions are required to be equal:

$$
D_{\mathrm{NS}}^{\mathrm{A}}=-12 \pi^{2} Q^{2} \frac{\mathrm{d} \Pi_{\mathrm{T}}^{\mathrm{A}}}{\mathrm{d} Q^{2}}=-12 \pi^{2} Q^{2} \frac{\mathrm{d} \Pi_{\mathrm{T}}^{\mathrm{V}}}{\mathrm{d} Q^{2}}=D_{\mathrm{NS}}^{\mathrm{V}}=N_{\mathrm{C}}\left(1+3 C_{\mathrm{F}} \bar{a}_{\mathrm{s}}+O\left(\bar{a}_{\mathrm{s}}^{2}\right)\right) .
$$

In [17] it was shown that the equality of $D_{\mathrm{NS}}^{\mathrm{A}}$ and $D_{\mathrm{NS}}^{\mathrm{V}}$, at the two-loop level, requires the leading-order renormalization $Z_{\mathrm{A}}=1-4 C_{\mathrm{F}} a_{\mathrm{s}}+O\left(a_{\mathrm{s}}^{2}\right)$, which then gives an infinite NLO renormalization in (13), with $Z_{2,1}=-2 \beta_{1} C_{\mathrm{F}}$, according to (15). We now investigate the situation, to all orders in the coupling $N_{\mathrm{F}} a_{\mathrm{s}}$, in the large- $N_{\mathrm{F}}$ limit.

As $N_{\mathrm{F}} \rightarrow \infty$, with $N_{\mathrm{F}} \bar{a}_{\mathrm{s}}$ fixed, all radiative corrections to the parton model are suppressed by at least one factor of $1 / N_{\mathrm{F}}$. From the $O\left(1 / N_{\mathrm{F}}\right)$ corrections to $D_{\mathrm{NS}}^{\mathrm{V}}$, we obtain those in

$$
C_{\mathrm{R}} \equiv \frac{D^{\mathrm{V}}}{N_{\mathrm{F}} N_{\mathrm{C}}}=\frac{D_{\mathrm{NS}}^{\mathrm{V}}}{N_{\mathrm{C}}}+O\left(1 / N_{\mathrm{F}}^{2}\right)=1+\frac{C_{\mathrm{F}}}{T_{\mathrm{F}} N_{\mathrm{F}}} \sum_{n=1}^{\infty} R_{n}\left(T_{\mathrm{F}} N_{\mathrm{F}} \bar{a}_{\mathrm{s}}\right)^{n}+O\left(1 / N_{\mathrm{F}}^{2}\right),
$$

in the $\overline{\mathrm{MS}}$ scheme, with $O\left(1 / N_{\mathrm{F}}\right)$ coefficients given in closed form by

$$
R_{n}=\frac{3}{2} 4^{n}(n-1) ! \sum_{p=1}^{n} \frac{\left(-\frac{5}{9}\right)^{n-p}}{(n-p) !} \frac{\Psi_{p+1}^{[p]}}{(p-1) !}
$$

in terms of the recently obtained momentum-scheme coefficients [20]

$$
\Psi_{n}^{[n-1]}=\frac{(n-1) !}{(-3)^{n-1}}\left[-2 n+4-\frac{n+4}{2^{n}}+\frac{16}{n-1} \sum_{n / 2>s>0} s\left(1-2^{-2 s}\right)\left(1-2^{2 s-n}\right) \zeta_{2 s+1}\right],
$$

that specify the $O\left(1 / N_{\mathrm{F}}\right)$ terms of $\Psi \equiv \beta_{\mathrm{MOM}}$, the QED $\beta$-function in the momentum $(\mathrm{MOM})$ subtraction scheme. The all-orders result (24) reproduces the $O\left(1 / N_{\mathrm{F}}\right)$ 4-loop results of $[13$, for $n \leq 4$. To transform to the $\overline{\mathrm{MS}}$ Adler-function coefficients (23), one has merely to observe that, at $O\left(1 / N_{\mathrm{F}}\right)$, a MOM-scheme subtraction at $-q^{2}=Q^{2}$ is equivalent to a $\overline{\mathrm{MS}}$ renormalization at $\mu^{2}=\mathrm{e}^{-5 / 3} Q^{2}$. From (23,24), we readily obtain the results for $R_{n}$ in Table 2 . The first 3 coefficients agree with [7]; the remainder are new.

\subsection{Axial renormalization constant}

We determine the renormalization constant of (13), at $O\left(1 / N_{\mathrm{F}}\right)$, by imposing the chiralsymmetry relations $D_{\mathrm{NS}}^{\mathrm{A}}=D_{\mathrm{NS}}^{\mathrm{V}}$ and $\mathrm{d} \Pi_{\mathrm{L}}^{\mathrm{A}} / \mathrm{d} Q^{2}=0$. First we calculate the contributions 
to the axial correlator of the generic $n$-loop bare diagrams with $n-1$ quark loops, keeping $n$ as an algebraic variable. This result involves an $F_{3,2}$ hypergeometric function, whose expansion about $\varepsilon=0$ cannot be effected in terms of $\zeta$-functions. Fortunately, couplingconstant renormalization ensures that one needs the function only in the limit [20] $\varepsilon \rightarrow 0$, with $n \varepsilon$ fixed, where it is a tri-gamma function whose expansion yields $\zeta$-functions [21]. Analyzing the residual, analytically simpler, bare contributions, we encounter the 3 anticipated problems that must be solved by the non-minimal renormalization (13): there are non-subtractable singular bare terms, involving $\ln \left(Q^{2} / \mu^{2}\right) / \varepsilon$; the bare transverse axial and vector contributions differ by logarithmic terms; the bare longitudinal axial contributions also have a logarithmic $Q^{2}$-dependence.

For these problems, a single cure is available: the renormalization (13), which acts only on the one-loop term, at $O\left(1 / N_{\mathrm{F}}\right)$. At $n+1$ loops, only one new constant is at our disposal: the leading term in the large- $N_{\mathrm{F}}$ expansion of the coefficient $Z_{n, 0}$. Following the methods of [20], we have explicitly verified that this suffices to solve all 3 problems. The required all-orders solution to (14) is

$$
Z_{\mathrm{A}}=1+\frac{C_{\mathrm{F}} \varepsilon}{6 T_{\mathrm{F}} N_{\mathrm{F}}} \widehat{\mathrm{L}}\left\{\frac{\ln \left(1-\frac{4}{3} T_{\mathrm{F}} N_{\mathrm{F}} a_{\mathrm{s}} / \varepsilon\right)}{B(2-\varepsilon, 2-\varepsilon) B(3-\varepsilon, 1+\varepsilon)}\right\}+O\left(1 / N_{\mathrm{F}}^{2}\right),
$$

where $\widehat{\mathrm{L}}$ is the Laurent operator, which removes non-singular terms from the perturbative expansion of the term in braces, in accordance with the Ansatz of (13). Note that the Euler $B$-functions, in (25), result from the residue at $n=1$ of the analytical expression for the bare $n$-loop contribution, in much the same way that the $O\left(1 / N_{\mathrm{F}}\right)$ QED $\beta$-function of any MS-like scheme results from a residue at $n=0$ [20, 22]. Expanding (25) to order $a_{\mathrm{s}}^{3}$, we verify the large- $N_{\mathrm{F}}$ terms in the axial-current renormalization used in [6]. Using it to all orders, we verify the chiral-symmetry relations $D_{\mathrm{NS}}^{\mathrm{A}}=D_{\mathrm{NS}}^{\mathrm{V}}$ and $\mathrm{d} \Pi_{\mathrm{L}}^{\mathrm{A}} / \mathrm{d} Q^{2}=0$, at $O\left(1 / N_{\mathrm{F}}\right)$ in the $\overline{\mathrm{MS}}$ scheme.

\subsection{Deep-inelastic processes}

We now calculate all the $O\left(1 / N_{\mathrm{F}}\right)$ radiative corrections to the sum rules (1, 21), which differ only by terms of $O\left(1 / N_{\mathrm{F}}^{2}\right)$. For the polarized deep-inelastic electron scattering sum rule (2), we calculate the generic $n$-loop $O\left(1 / N_{\mathrm{F}}\right)$ bare diagrams for forward Compton scattering of a vector current, with momentum $q$, off a zero-momentum quark [6, 11, 23], by inserting $n-1$ quark loops into the one-loop diagrams, obtaining simple $\Gamma$-functions. The Compton diagrams must then be divided [6] by (25), to obtain the coefficient $C_{\mathrm{Bjp}}$ of the axial current (13) in the OPE of $V V$. After the coupling-constant renormalization

$$
\left(\frac{g_{0}}{4 \pi}\right)^{2}=\left(\frac{\mu^{2} \mathrm{e}^{\gamma}}{4 \pi}\right)^{\varepsilon} \frac{a_{\mathrm{s}}}{1-\frac{4}{3} T_{\mathrm{F}} N_{\mathrm{F}} a_{\mathrm{s}} / \varepsilon}+O\left(1 / N_{\mathrm{F}}^{2}\right)
$$

we obtain the $O\left(1 / N_{\mathrm{F}}\right)$ contributions to

$$
C_{\mathrm{K}} \equiv C_{\mathrm{GLS}}=C_{\mathrm{Bjp}}+O\left(1 / N_{\mathrm{F}}^{2}\right)=1+\frac{C_{\mathrm{F}}}{T_{\mathrm{F}} N_{\mathrm{F}}} \sum_{n=1}^{\infty} K_{n}\left(T_{\mathrm{F}} N_{\mathrm{F}} \bar{a}_{\mathrm{s}}\right)^{n}+O\left(1 / N_{\mathrm{F}}^{2}\right),
$$

in the $\overline{\mathrm{MS}}$ scheme, with $O\left(1 / N_{\mathrm{F}}\right)$ coefficients given in closed form by

$$
K_{n}=\lim _{z \rightarrow 0}\left(-\frac{4}{3} \frac{\mathrm{d}}{\mathrm{d} z}\right)^{n-1} \bar{K}(z), \quad \bar{K}(z)=-\frac{(3+z) \exp (5 z / 3)}{\left(1-z^{2}\right)\left(1-z^{2} / 4\right)},
$$


where $\bar{K}(z)$ is obtained from the $\varepsilon \rightarrow 0$ limit of the bare $n$-loop contribution, with $z=n \varepsilon$ fixed. The renormalization constant (25) precisely cancels the infinities of the bare terms, obtained from the residue of the pole at $n=0$. The same results are obtained for $C_{\mathrm{GLS}}$, in the large- $N_{\mathrm{F}}$ limit, since the diagrams that distinguish the sum rules have three (or more) gluons in the $t$-channel [6] and hence are (at least) of order $1 / N_{\mathrm{F}}^{2}$.

From (28), we readily obtain the results for $K_{n}$ in Table 2 . The first 3 coefficients agree with [6]; the remainder are new. Combining $(23,28)$, we obtain the $O\left(1 / N_{\mathrm{F}}\right) \overline{\mathrm{MS}}$ coefficients $S_{n}=\frac{3}{4}\left(K_{n+1}+R_{n+1}\right)$ in (12), also given in Table 2, which may be extended, ad libitum. The first 2 coefficients, $S_{1}$ and $S_{2}$, agree with (6); the remainder are new.

\section{Conclusions}

We have discovered the 7 intricate connections of (10,11), between the highly non-trivial NNLO radiative corrections, $C_{\mathrm{K}}$ and $C_{\mathrm{R}}$, to the GLS sum rule [6] and the Adler function [7] of the flavour-singlet vector current, given in Table 1 . Forming $\Delta_{\mathrm{S}} \equiv\left(C_{\mathrm{K}} C_{\mathrm{R}}-1\right)$, we find the remarkable result of (5), namely a linear function of the coupling, multiplying the two-loop $\beta$-function, and hence reducing from 11 to 4 the number of independent colour structures in $\Delta_{\mathrm{S}}$, up to $O\left(\bar{a}_{\mathrm{s}}^{3}\right)$. Two of the 4 coefficients in (间), namely $S_{1}$ and $S_{2}$, have been obtained $a b$ initio, as the first two members of the series of large- $N_{\mathrm{F}}$ coefficients in (12), whose higher-order members can be obtained from our new all-orders results (23,28). Values are given in Table 2 for $n<10$.

The consistency of the prescription (17) with chiral symmetry has been demonstrated, in the large- $N_{\mathrm{F}}$ limit, using the all-orders axial renormalization constant (25). A validation of $S_{\mathrm{A}}$ and $S_{\mathrm{F}}$, the $O\left(1 / N_{\mathrm{F}}^{2}\right)$ coefficients in (5), has not been attempted here. We note, however, that recent progress with $O\left(1 / N_{\mathrm{F}}^{2}\right)$ corrections to QED [24], and to the Gross-Neveu model [25], suggests that one may eventually be able to obtain the entire series of coefficients that have $S_{\mathrm{A}}$ and $S_{\mathrm{F}}$ as their leading members. In any case, the relations (10,11) give one a high degree of confidence in the accuracy of [6, , ]. Section 3 offers some general observations, suggesting that the relation $\Delta_{\mathrm{S}} \propto \beta$ may persist beyond NNLO. In any case, we confidently expect the expansion of $\Delta_{\mathrm{S}}$ to involve only the coefficients of the $\beta$-function, multiplied by linear combinations of colour factors.

We believe that the hypothesis $\Delta_{\mathrm{S}} \propto \beta$ merits close attention. Its proof (or disproof) could contribute significantly to an area of field theory that combines deep principles [1, 10] with calculational achievement [6, 7, 18, 26] and phenomenological relevance [27]. We recommend careful re-examination of the Crewther connection [1] in the light of our findings. The new connections (5,8) suggest that gauge theories know much more about it than has been supposed.

Acknowledgments We thank Bob Jaffe for asking us about the status of [1] in QCD. ALK thanks Peter White for help in organizing his recent fruitful tour in England, contributing to the appearance of this work, which is dedicated to the memory of his late friend and colleague S. G. Gorishny, who mentioned to him, in private discussions in 1987, not long before his death from cancer, that "calculations of the NNLO corrections to the GLS sum rule may be connected to problems of the manifestation of the anomaly and of the careful treatment of its renormalization within dimensional regularization". 
Table 1 NNLO results of [6, 7] for $C_{\mathrm{K}}=\sum_{n} k_{n} T_{n}+O\left(\bar{a}_{\mathrm{s}}^{4}\right), C_{\mathrm{R}}=\sum_{n} r_{n} T_{n}+O\left(\bar{a}_{\mathrm{s}}^{4}\right)$.

\begin{tabular}{rrll}
$n$ & $T_{n}$ & $k_{n}$ & $r_{n}$ \\
\hline 1 & $C_{\mathrm{F}} \bar{a}_{\mathrm{s}}$ & -3 & 3 \\
\hline 2 & $C_{\mathrm{F}}^{2} \bar{a}_{\mathrm{s}}^{2}$ & $\frac{21}{2}$ & $-\frac{3}{2}$ \\
3 & $C_{\mathrm{F}} C_{\mathrm{A}} \bar{a}_{\mathrm{s}}^{2}$ & -23 & $\frac{123}{2}-44 \zeta_{3}$ \\
4 & $C_{\mathrm{F}} T_{\mathrm{F}} N_{\mathrm{F}} \bar{a}_{\mathrm{s}}^{2}$ & 8 & $-22+16 \zeta_{3}$ \\
\hline 5 & $C_{\mathrm{F}}^{3} \bar{a}_{\mathrm{s}}^{3}$ & $-\frac{3}{2}$ & $-\frac{69}{2}$ \\
6 & $C_{\mathrm{F}}^{2} C_{\mathrm{A}} \bar{a}_{\mathrm{s}}^{3}$ & $\frac{1241}{9}-\frac{176}{3} \zeta_{3}$ & $-127-572 \zeta_{3}+880 \zeta_{5}$ \\
7 & $C_{\mathrm{F}} C_{\mathrm{A}}^{2} \bar{a}_{\mathrm{s}}^{3}$ & $-\frac{10874}{27}+\frac{440}{3} \zeta_{5}$ & $\frac{90445}{54}-\frac{10948}{9} \zeta_{3}-\frac{440}{3} \zeta_{5}$ \\
8 & $C_{\mathrm{F}}^{2} T_{\mathrm{F}} N_{\mathrm{F}} \bar{a}_{\mathrm{s}}^{3}$ & $-\frac{133}{9}-\frac{80}{3} \zeta_{3}$ & $-29+304 \zeta_{3}-320 \zeta_{5}$ \\
9 & $C_{\mathrm{F}} C_{\mathrm{A}} T_{\mathrm{F}} N_{\mathrm{F}} \bar{a}_{\mathrm{s}}^{3}$ & $\frac{7070}{27}+48 \zeta_{3}-\frac{160}{3} \zeta_{5}$ & $-\frac{31040}{27}+\frac{7168}{9} \zeta_{3}+\frac{160}{3} \zeta_{5}$ \\
10 & $C_{\mathrm{F}} T_{\mathrm{F}}^{2} N_{\mathrm{F}}^{2} \bar{a}_{\mathrm{s}}^{3}$ & $-\frac{920}{27}$ & $\frac{4832}{27}-\frac{1216}{9} \zeta_{3}$ \\
11 & $\frac{N_{\mathrm{F}}}{N_{\mathrm{C}}} d_{a b c}^{2} \bar{a}_{\mathrm{s}}^{3}$ & $-\frac{11}{3}+8 \zeta_{3}$ & $\frac{11}{3}-8 \zeta_{3}$ \\
\hline
\end{tabular}

Table 2 Large- $N_{\mathrm{F}}$ expansions (22,27, 12), obtained from (23, 28), with $x \equiv T_{\mathrm{F}} N_{\mathrm{F}} \bar{a}_{\mathrm{s}}$.

$$
\begin{aligned}
\sum_{n} & <10 R_{n} x^{n}=3 x+\left[-22+16 \zeta_{3}\right] x^{2}+\left[\frac{4832}{27}-\frac{1216}{9} \zeta_{3}\right] x^{3}+\left[-\frac{392384}{243}+\frac{25984}{27} \zeta_{3}+\frac{1280}{3} \zeta_{5}\right] x^{4} \\
& +\left[\frac{11758720}{729}-\frac{5073920}{729} \zeta_{3}-\frac{194560}{27} \zeta_{5}\right] x^{5}+\left[-\frac{3499697920}{19683}+\frac{357201920}{6561} \zeta_{3}+\frac{20787200}{243} \zeta_{5}+\frac{71680}{3} \zeta_{7}\right] x^{6} \\
& +\left[\frac{381559797760}{177147}-\frac{9308446720}{19683} \zeta_{3}-\frac{2029568000}{2187} \zeta_{5}-\frac{5447680}{9} \zeta_{7}\right] x^{7} \\
& +\left[-\frac{5056220794880}{177147}+\frac{2445582254080}{531441} \zeta_{3}+\frac{200033075200}{19683} \zeta_{5}+\frac{814858240}{81} \zeta_{7}+\frac{194969600}{81} \zeta_{9}\right] x^{8} \\
& +\left[\frac{5908327309475840}{14348907}-\frac{239732713062400}{4782969} \zeta_{3}-\frac{20850920652800}{177147} \zeta_{5}-\frac{318236262400}{2187} \zeta_{7}-\frac{59270758400}{729} \zeta_{9}\right] x^{9} \\
\hline \sum_{n} & <10 K_{n} x^{n}=-3 x+8 x^{2}-\frac{920}{27} x^{3}+\frac{38720}{243} x^{4}-\frac{238976}{243} x^{5}+\frac{130862080}{19683} x^{6}-\frac{10038092800}{177147} x^{7} \\
& +\frac{274593587200}{531441} x^{8}-\frac{8251909947920}{14348907} x^{9} \\
\hline \sum_{n} & <10 S_{n} x^{n}=\left[-\frac{21}{2}+12 \zeta_{3}\right] x+\left[\frac{326}{3}-\frac{304}{3} \zeta_{3}\right] x^{2}+\left[-\frac{9824}{9}+\frac{6496}{9} \zeta_{3}+320 \zeta_{5}\right] x^{3} \\
+ & {\left[\frac{2760448}{243}-\frac{1268480}{243} \zeta_{3}-\frac{48640}{9} \zeta_{5}\right] x^{4}+\left[-\frac{280736320}{2187}+\frac{89300480}{2187} \zeta_{3}+\frac{5196800}{81} \zeta_{5}+17920 \zeta_{7}\right] x^{5} } \\
+ & {\left[\frac{10320047360}{6561}-\frac{2327111680}{6561} \zeta_{3}-\frac{507392000}{729} \zeta_{5}-\frac{1361920}{3} \zeta_{7}\right] x^{6} } \\
+ & {\left[-\frac{3723517199360}{177147}+\frac{611395563520}{177147} \zeta_{3}+\frac{50008268800}{6561} \zeta_{5}+\frac{203714560}{27} \zeta_{7}+\frac{48742400}{27} \zeta_{9}\right] x^{7} } \\
+ & {\left[\frac{485484017500160}{1594323}-\frac{59933178265600}{1594323} \zeta_{3}-\frac{5212730163200}{59049} \zeta_{5}-\frac{79559065600}{729} \zeta_{7}-\frac{14817689600}{243} \zeta_{9}\right] x^{8} } \\
+ & {\left[-\frac{7616109282344960}{1594323}+\frac{726735764193280}{1594323} \zeta_{3}+\frac{195646580326400}{177147} \zeta_{5}+\frac{1120185221120}{729} \zeta_{7}\right.} \\
+ & \left.\frac{316630630400}{243} \zeta_{9}+\frac{7821721600}{27} \zeta_{11}\right] x^{9}
\end{aligned}
$$




\section{References}

[1] R. J. Crewther, Phys. Rev. Lett. 28 (1972) 1421.

[2] S. L. Adler, Phys. Rev. 177 (1969) 2426;

J. S. Bell and R. Jackiw, Nuovo Cimento 60A (1969) 47.

[3] J. D. Bjorken, Phys. Rev. 148 (1966) 1467; D1 (1970) 1376.

[4] S. L. Adler, Phys. Rev. D10 (1974) 3714.

[5] D. J. Gross and C. H. Llewellyn Smith, Nucl. Phys. B14 (1969) 337.

[6] S. A. Larin and J. A. M. Vermaseren, Phys. Lett. B259 (1991) 345.

[7] S. G. Gorishny, A. L. Kataev and S. A. Larin, Phys. Lett. B259 (1991) 144;

Pisma ZhETF 53 (1991) 121.

[8] B. A. Kniehl and J. H. Kühn, Nucl. Phys. B329 (1990) 547.

[9] K. G. Chetyrkin and A. Kwiatkowski, Phys. Lett. B305 (1993) 285.

[10] S. L. Adler and W. Bardeen, Phys. Rev. 182 (1969) 1517.

[11] S. G. Gorishny and S. A. Larin, Phys. Lett. B172 (1986) 109.

[12] K. G. Chetyrkin, A. L. Kataev and F. V. Tkachov, Phys. Lett. B85 (1979) 277;

M. Dine and J. Sapirstein, Phys. Rev. Lett. 43 (1979) 668;

W. Celmaster and R. Gonsalves, Phys. Rev. Lett. 44 (1980) 560.

[13] S. G. Gorishny, A. L. Kataev, S. A. Larin and L. R. Surguladze, Phys. Lett. B256 (1991) 81.

[14] S. A. Larin, Phys. Lett. B303 (1993) 113.

[15] R. Akhoury and S. Titard, Univ. Michigan preprint UM-TH-91-21 (1991);

G. T. Gabadadze and A. A. Pivovarov, Pisma ZhETF 54 (1991) 305;

Yad. Fiz. 56 (1993) 257;

M. Bos, UCLA preprint UCLA-92-TEP-41 (1992).

[16] G. 't Hooft and M. Veltman, Nucl. Phys. B44 (1972) 189;

D. A. Akyeampong and R. Delburgo, Nuovo Cimento 17A (1973) 578;

P. Breitenlohner and D. Maison, Comm. Math. Phys. 52 (1977) 11.

[17] I. Antoniadis, Phys. Lett. B84 (1979) 223;

T. L. Trueman, Phys. Lett. B88 (1979) 331.

[18] E. B. Zijlstra and W. L. van Neerven, Phys. Lett. B297 (1992) 377.

[19] D. Kreimer, Phys. Lett. B237 (1990) 59;

P. A. Baikov and V. A. Ilyin, Teor. Mat. Fiz. 88 (1991) 163.

[20] D. J. Broadhurst, Z. Phys. C58 (1993) 339. 
[21] D. J. Broadhurst, Z. Phys. C32 (1986) 249;

D. T. Barfoot and D. J. Broadhurst, Z. Phys. C41 (1988) 81.

[22] A. Palanques-Mestre and P. Pascual, Comm. Math. Phys. 95 (1984) 277.

[23] S. G. Gorishny, S. A. Larin and F. V. Tkachov, Phys. Lett. B124 (1983) 217;

S. G. Gorishny and S. A. Larin, Nucl. Phys. B283 (1987) 452.

[24] J. A. Gracey, Mod. Phys. Lett. A7 (1992) 1945.

[25] J. A. Gracey, Liverpool preprint LTH-312 (1993);

S. E. Derkachov, N. A. Kivel, A. S. Stepanenko and A. N. Vasil'ev, Saclay preprint SPHT-93-016 (1993).

[26] D. J. Broadhurst, A. L. Kataev and O. V. Tarasov, Phys. Lett. B298 (1993) 445; T. Kinoshita, Phys. Rev. D47 (1993) 5013

[27] J. Chýla, A. L. Kataev and S. A. Larin, Phys. Lett. B267 (1991) 269;

J. Chýla and A. L. Kataev, Phys. Lett. B297 (1992) 385;

E. Braaten, S. Narison and A. Pich, Nucl. Phys. B373 (1992) 581. 